\title{
The Modern Treatment of Leprosy.
}

R. G. Cochrane.

Being a Paper given before the Tropical Medicine Section at the Centenary Meeting of the British Medical Association, held in London, fuly, 1932.

TE subject which has been chosen is one of very great interest, and because the time at my disposal is relatively short, I shall only be able to deal with it in its essentially practical aspects, omitting any academic discussions.

The first question to decide when one is faced with the problem of the treatment of a disease like leprosy, is whether the case is one which will benefit from treatment. Modern developments have indicated that this disease tends to be self-healing. In the earlier years of the more recent treatments, stress was laid on the fact that many advanced cases were no longer dangerous to the public, because the disease had become naturally arrested, leaving signs (comparable to the pock-marks of smallpox) that the patient had suffered from leprosy, and that the disease had only left stigmata of its presence.

It has been borne upon me forcibly, especially as the result of work in Africa, that as in tuberculosis, so in leprosy, the disease may become arrested at any point ${ }^{1}$. One sees many instances of healthy adults with one or two isolated patches indicative of a leprotic condition, but which have remained stationary for years. In other words, they are selfarrested cases in the early stage, leaving the solitary scar of evidence of an infection which has died out. This aspect is of much importance to workers, for it is just as futile to treat the early arrested case as the late. In passing, I should say that no child or adolescent should be assumed to be in this stage without prolonged observation, but adults who have had early signs of the disease for a period of years do not necessarily need active treatment. Therefore, before one can discuss treatment it seems essential to lay down the signs of activity, and these were set down at the Manila Conference ${ }^{2}(1931)$, as follows :-

Signs of Activity.

"Active cases are those in which there are clinical or microscopical evidences of progressive or of recessive changes in lesions, with or without accompanying systemic disturbances. These evidences include the following: positive bacteriological findings in skin or mucous mem- 
brane determined by the usual methods; the presence of raised or erythematous lesions ; increase or diminution of lesions in size or number; tenderness of nerves, with or without thickening."

Having determined the point that a given case is an active one, then the question of treatment arises.

It is axiomatic to state that before one can treat leprosy with any hope of success it is essential to treat any other disease or cause of debility in the patient. One, therefore, assumes that the patient has been examined for the possible existence of the various predisposing causes, and these have been or are being, treated efficiently.

The modern treatment of leprosy from the initial researches of Heiser, Rogers and others, has been based on the assumption that in hydnocarpus (chaulmoogra) oil, there is something which acts in a special manner, even though it is not generally held that it is a true specific for the disease. Just how this remedy acts can only be surmised, but the fact remains that no physician would be willing to do without the hydnocarpus preparations in the treatment of leprosy. The various preparations which can be used in order of preference are :-

(1) Iodised or creosoted esters.

(2) Alepol. (This is a special variety of sodium hydnocarpate of the lower melting point fatty acids.)

(3) Plain oil with creosote.

The choice of the remedy is chiefly a matter of expense and ease of obtaining it, and, therefore, in this paper, I shall deal briefly with the ordinary routine treatment of a case of leprosy, and then touch on the question of the important complication known as lepra-reaction.

If the case is an early pure nerve case, the procedure would be to inject subcutaneously or intramuscularly either 4 per cent. solution of alepol, or the ethyl esters of the oil. The choice of the remedy in such a case is not so important. The dosage to commence with is about 2 c.cs., going up by weekly injections to 12 c.cs. It will be found that it is inadvisable to increase the dosage above 12 c.cs. of a 6 per cent. solution of alepol, or 12 c.cs. of the oil or esters. I am aware that certain authorities state that it is not necessary to give more than 5 c.cs., but I think everything tends to show that the more of the remedy one can give within the above limits the better.

In addition to this general treatment, if the patient has erythematous or hypopigmented patches, these require local treatment. Erythematous lesions should be injected 
intradermally. This method will be described later. Hypopigmented patches should be painted with trichloracetic acid. The latter is applied in strength of $1: 5$ for the face or $1: 3$ for the body. Care must be taken to apply it carefully or scarring and kcloid formation may result. If the patient suffers from nerve pain then ephredine ${ }^{3}\left(\frac{1}{2}\right.$ gr. in 1-oz. of water) or adrenalin, plus an alkaline (bicarbonate) mixture should be prescribed. If pain is severe, stripping the nerve sheath may be necessary. Morphia or opium should not be given unless unavoidable. Recently dilo oil (calophyllum bigator) has been tried in $\mathrm{Fiji}^{4}$ with remarkable success. The method of controlling the dosage of the drugs will be described shortly.

Cutaneous Leprosy.

This, generally speaking, is much more difficult to treat, and if the case is at all advanced, the ultimate prognosis is poor. Lowe ${ }^{5}$, of Dichpali, has recently sounded a timely warning against unreasoned optimism.

The cutaneous case is usually taken to be one in which bacilli can be demonstrated by the usual methods, and is treated along the following lines :-

(1) Local.

(2) General.

(3) Treatment of complications.

Again the drug of choice is the esters; the method of choice is intradermal injections. For this a special needle with a guard $3 \mathrm{~mm}$. from the point is used. The technique is as follows :-

An all-glass or record syringe is used with a fine needle fitted with a guard about three $\mathrm{mm}$. from the point. This prevents the needle penetrating too far into the tissue. The skin having previously been painted with iodine the needle is then introduced into and not under the skin. The thickness of the skin varies in different parts of the body ; where it is thin, the needle is introduced in a sloping manner, so as to form an acute angle with the skin. If it is thicker, then it is preferable to insert it at right angles. With a little practice, the technique is soon mastered and one finds little difficulty in injecting into the corium and not into the subcutaneous tissues. An amount of the drug is injected so as to raise a wheal of about one-third of an inch in diameter. If a large area is to be injected, it is completely infiltrated so as to produce coalescing "injection wheals." Some forty punctures are necessary to inject about 6 c.cs. of the esters. It has been suggested that not more than 5 c.cs. 
should be injected intradermally. If a larger quantity than this is given, or if there are only a few lesions to be - injected, then the balance can be given subcutaneously or intramuscularly. It has been the experience of leprosy workers for many decades that the case which can take large doses of chaulmoogra oil by mouth generally improves. I feel certain that while intradermal injections are of very great value, the remedy is absorbed so slowly that the general effect of the drug does not begin to be experienced for some little while, although the advantage of this method is that there appears to be an accumulative effect due to its slow absorption. In one case I am at present treating, I follow a routine of intradermal, subcutaneous and oral administration. The oil can be obtained in such a purified form that I find that even the most sensitive gastric mucosa will tolerate it ; but it must be obtained from a firm which specially prepares the remedy.

The following, then, is a suggested routine for cutaneous cases. Weekly injections of iodised esters intradermally or subcutaneously. It is wise to give two or three week's rest after reaching the maximum dosage, and during this period if desired, hydnocarpus oil can be given by mouth. The initial amount advised is $2 \mathrm{mms}$., going up to a teaspoonful or more, according to the patient's tolerance. In addition, if there are a large number of lesions, it is well to paint those lesions which have not been injected with trichloracetic acid.

If the patient shows any signs of reaction or complains of weakness and lethargy, the injections or oral treatment should be stopped and a complete rest given and a tonic prescribed.

I have recently compared iodised with creosoted esters, and I find that the creosoted esters are considerably more painful and that the average patient in this country will not stand the injections for long, whereas iodised esters can be given with comparatively little pain.

With regard to the other remedies, many workers prefer alepol. This is cheaper and effective, but for intradermal use it is absorbed too rapidly for it to give permanent results. The optimum strength is a 4 per cent. solution, increasing gradually to a maximum of 12 c.cs. The initial dosage of anti-leprotic remedies in cutaneous cases is $\frac{1}{2}$ to 1 c.c., increasing by $\frac{1}{2}$ c.cs until a maximum of 12 c.cs is reached. In nerve cases the initial dose may be as high as 2 c.cs., increasing to 12 c.cs.

The controlling of the injections is a very important 
matter, and the following are the indications that the dosage should not be increased.

(l) Appearance of fresh eruptions, especially the socalled rose-red nodules.

(2) A swinging of the temperature. This variation in temperature may be both above as well as below the normal line. In fact, I believe in mild lepra reaction there may be what I term a pyrexia below the normal line, that is, an irregular variation below the ordinary accepted normal temperature of $98 \cdot 4$.

(3) Rise in sedimentation index.

(4) Feeling of general debility with loss of weight.

(5) Breaking down and discharging lesions without apparent rise of temperature, though there is usually a subnormal variation.

Tuberculoid leprosy, which has recently been described by Wade ${ }^{6}$, is particularly suitable for intradermal treatment.

\section{Treatment of Complications.}

1. Lepra-reaction. This has been defined as a state or condition of the body produced by the breaking down of leprotic foci. It is very important to recognise lepra-reaction in the very early stages, and in this connection sufficient importance has not been paid to the temperature chart. Recently the question of normal temperature has been raised in medical papers In leprosy it is of utmost importance to take note of variations in the temperature chart below the normal line, as this may indicate commencing activity. If the patient is improving it is not necessary to reduce dosage, but if there are continuous variations below the normal line it is indicative of something unusual taking place, and treatments should be proceeded with cautiously. The temperature may rise without a corresponding rise in the sedimentation index test, indicating that this may be a more delicate sign than the S.I. test.

The treatment of lepra reaction is the stopping of injections, the giving of a purge and putting the patient on a bland diet, and the administration of Pot. Ant. Tartrate $0 \cdot 02-0.04$ every other day. Lepra-reaction may proceed for a very long time and nothing seem of any avail in reducing the fever. Recently 3 c.cs. of a 1 per cent. solution of Mercurochrome soluble 220 has been given intravenously for this condition with apparent improvement.

2. Eye Reactions. Generally speaking leprosy of the eye goes on progressively until blindness ensues. Complete loss of vision can be stayed for a very long time, and if 
general treatment is cautiously undertaken, watching carefully for any flare up, no damage need be done. The local treatment is that of frequent bathing with an alkaline lotion. Atropine or hyoscine drops and ointment should also be used.

Before I close, reference should be made to some other remedies used. The gold preparations have, I am certain, a limited place in treatment. The tendency is to use too large a dosage and continue too long. I personally prefer Solganol B given intramuscularly in the following weekly dosage :-

First and second weeks

Third and fourth weeks

Fifth and sixth weeks ...

$\begin{array}{cccc}\ldots & \ldots & 0.01 & \text { grams } \\ \ldots & \ldots & 0.05 & , \\ \ldots & \ldots & 0.01 & \text { ", }\end{array}$

In using heavy metals it should be remembered that generally speaking small doses may be beneficial, large doses harmful.

The indications for gold therapy are :-

(1) The presence of leprotic affection of the eyes.

(2) The production of lepra fever by the usual methods of treatment.

Not more than two courses, with 21 days rest, should be given without a prolonged interval (three months) for fear of starting a reaction. One should remember that if a patient has had much fever he needs building up, and no drug should be given which tends to break down leprotic foci. Muir ${ }^{7}$ has recently advised against gold treatment, but workers in Malaya ${ }^{8}$ are confirming Hoffman's ${ }^{9}$ view that it is of definite value.

I must apologise for dealing so superficially with this subject, but I have, in the time at my disposal, tried to indicate the general lines along which treatment should proceed. To-day the prognosis in leprosy is being more accurately evaluated, and the extreme optimism of the early days of the new treatment is being replaced by a more reasonable view, while the complete pessimism of the older workers is gradually being replaced by a brighter outlook. While admitting that there is much room for improvement, yet it can be said that the modern therapeutics of leprosy has altered completely the whole situation, and has brought relief permanently to many hundreds of sufferers, and made existence reasonable and comfortable for thousands to whom life otherwise would be but a living death. To have attained this in a matter of a few years is indeed an achievement, and 
one looks forward to further advances as the study of this long neglected disease is taken up more extensively.

\section{REFERENCES.}

(1) Cochrane, R. G.- "Leprosy, its Prevention and Control." Fournal of State Medicine, Vol. xxxix, No. 10.

(2) Report of Leonard Wood Memorial Conference. Philippine Fournal of Science, Vol. 44, No. 4, Apr., 1931. p. 463.

(3) Cochrane, R. G.- "Action of Ephredine in Leprosy." Lancet, Sept. 14 th, 1929, p. 551.

(4) Annual Medical Health Report for Fiji for the year 1930, p. 59.

(5) Lowe, John.- "Leprosy in India." Ind. Med. Gazette. Apr., 1932, p. 208.

(6) Wade, H. W.- "Demonstration of S. African Tuberculoid Leprosy." Proc. of the Roy. Society of Medicine, Vol. xxv, May, 1932.

(7) Muir, E.-Leprosy in India, Jan., 1932, p. 7.

(8) Ryrie, G. A.-Private Communication.

(9) Hoffmann, W. H.- "Gold Treatment in Leprosy." Leprosy Review, Vol. 11, Apr., 1931, p. 43.

(10) Ryrie, G. A.-Private Communication.

(11) Muir, E., Chatterji, S. P.- "The Uses of Mercurochrome Soluble 220 in Leprosy." Leprosy in India, July, 1932, p. 4. 